\title{
Tangence
}

\section{La démolition de Pierre Marcelle ou le crime du signe}

\section{Margaret Cook}

Numéro 38, décembre 1992

Fiction policière et roman actuel

URI : https://id.erudit.org/iderudit/025738ar

DOI : https://doi.org/10.7202/025738ar

Aller au sommaire du numéro

Éditeur(s)

Tangence

ISSN

1189-4563 (imprimé)

1710-0305 (numérique)

Découvrir la revue

Citer cet article

Cook, M. (1992). La démolition de Pierre Marcelle ou le crime du signe.

Tangence, (38), 55-64. https://doi.org/10.7202/025738ar d'utilisation que vous pouvez consulter en ligne.

https://apropos.erudit.org/fr/usagers/politique-dutilisation/ 


\section{La démolition de Pierre Marcelle ou le crime du signe Margaret Cook}

Le roman policier attire l'écrivain contemporain qui se pose des questions sur la fausse innocence de l'écriture et du langage. L'écrivain réutilise les conventions du genre, et ces conventions servent à signaler le détachement d'un auteur commentant le processus même de l'écriture ou ironisant à ce sujet. Dans La démolition de Pierre Marcelle, publié chez Denoël en 1985, le crime et les termes qui s'y rattachent introduisent le lecteur dans l'univers du roman policier. Même si ce texte exploite d'autres conventions (comme celles du journal intime), ces formes servent de cadre à une réflexion sur le vrai et le faux, la vérité et le mensonge, ainsi qu'à un examen du langage.

La démolition met en scène la disparition supposée de Marianne et son absence constitue la raison d'être de l'action et du texte. Le narrateur bombe des citations d'auteurs, de cinéastes, de theoriciens et d'artistes sur les murs d'un chantier pour cerner sa propre expérience. Le texte s'ouvre par une citation suggestive de Walter Benjamin placée en exergue. Elle nous invite à contempler le "théâtre du crime", et affirme la nécessité de "découvrir la faute et [de] désigner le coupablen. En effet, qui dit crime dit coupable. Des photos auxquelles cette citation fait référence sont intercalées dans le texte, et la lecture doit tenir compte des concepts et des modèles du roman policier.

La présence d'un coupable présumé est révélée par la polysémie du vocabulaire qui renvoie aussi bien au domaine de la photo qu'à celui du crime: "shooter dans les décombres" (p. 16), le "déclencheur" (p. 52) et la "liste de contacts" (p. 57). Le chantier est désigné comme "lieu du crime "à deux reprises (p. 24 et 56). Un témoin (de mariage/du crime, p. 30), la loi (p. 43), ainsi que des "traces ", substantif qui réapparaît à quatre reprises (p. 16, 20, 27 et être "sur une piste*, p. 46), sont également évoqués. De fait, ce vocabulaire incite le lecteur à lire ce roman selon les règles du genre policier.

La structure du roman policier proposée par W.H. Auden est la suivante: fausse innocence, révélation de la culpabilité, faux 
repérage de la culpabilité, repérage de la vraie culpabilité, catharsis (arrestation), et véritable innocence ${ }^{1}$. La "mise à mort " (p. 67) de la cheminée et la *guillotine - (p. 34) deviennent autant d'éléments de la catharsis. Par ailleurs, les "témoignages" et le *greffier. (p. 73) font partie du repérage de la culpabilité. Au sujet de ce genre, Lichtenberg (une citation de lui fait partie des bombages dans La démolition) précise:

Celles [les maraudes] du roman policier ne sont-elles pas la quête, l'observation, la poursuite de toutes sortes d'indices, une véritable chasse aux indices qui constitue l'objet même des descriptions dans le roman. ${ }^{2}$

En effet, le narrateur veut *revenir avec un autre objectif pour attraper les détails* (p. 13) et il cherche «la faille* (p. 81), ce qui constitue un des éléments du rôle de détective.

Nous repérons également des personnages qui portent des masques cachant leur innocence ou leur culpabilité (donc des suspects): Jo B. (p. 10). Jacques A. (p. 35), Michel C. (p. 49), Thierry D. (p. 67), Olivier C. (p. 74), Pierre R. (p. 76), ainsi que Boubaker (p. 18) et Vilar (Vie-l'art? ou Vis l'art! p. 21). Dans le contexte du roman policier, les suspects contribuent au suspense, puisque l'un ou l'autre d'entre eux peut commettre un autre crime; la sécurité est impossible.

Nous observons aussi d'autres indices, notamment une *fenêtre dont les rideaux bougent sans cesse. (p. 14). Ce détail devient suspect simplement parce que le narrateur s'y attarde. Jean Cohen affirme que, dans le roman policier, le moindre objet devient suspect au même titre que tous les personnages; donc, tout l'espace romanesque est suspect, car sa structure est totalisante ${ }^{3}$. Néanmoins, dans ce cas, nous pouvons nous demander si nous ne sommes pas en présence de faux-semblants, d'un faux crime. En effet, les mots agissent sur l'esprit du lecteur et créent une atmosphère chargée de soupçons, mais l'innocence ou la culpabilité des personnages n'est jamais directement évoquée.

1 W.H. Auden, * Le presbytère coupable. Remarques sur le roman policier par un drogué. (1948), dans Uri Eisenzweig, Autopsies du roman policier, Paris, Union générale d'Editions, 1983, p. 115.

2 Lichtenberg, cité par Ernst Bloch, - Aspect philosophique du roman policier ", ibid., p. 252.

3 Jean Cohen, Le baut langage, Paris, Flammarion, 1979, p. 251. 
Aucune réponse ou solution claire n'est proposée malgré les questions posées: «Qui a tué? Qui ment? Quelle est l'histoire? Quelle est l'histoire?, c'est la vraie question* (p. 82). Autant de questions sans réponse qui attirent notre attention sur la difficulté de cerner «l'histoire*. Le lecteur est chargé de créer sa propre signification. Le secret reste secret; aucune révélation conventionnelle n'est faite. Robert Kroetsch remarque que, dans le roman canadien-anglais, la convention de suspense est menacée par l'idée du secret, car le suspense demande une révélation, or le voile du secret n'est pas levé 4 . Selon Philippe Sollers, tout roman contient une part des conventions du roman policier. Pour lui, toute intrigue tourne autour de la culpabilité 5 . Dans La démolition, un crime est dit, mais qu'en est-il de la culpabilité?

Moins prédominantes, d'autres conventions sont également exploitées dans La démolition. Elles peuvent fournir de nouveaux indices sur le mensonge et la vérité. La forme apparente du texte est celle du journal intime. Le texte se compose d'entrées, toutes datées (jour et mois), qui se suivent chronologiquement. Cependant, cette forme n'est pas strictement respectée. Il existe des analepses: le bombage «Et qui libre?* du 24 ou 25 mars n'est mentionné que le 30 mars (p. 42), ce qui est vraisemblable; mais il existe également des prolepses, et le narrateur affirme qu'il * anticipe un peu *. Cela implique que son entrée est écrite postérieurement à la date indiquée et qu'il triche donc avec le texte (pour brouiller les traces). Ces prolepses sont évidentes au début du récit:

Émergeant des ruines d'une usine de chaudronnerie - j'ai su plus tard qu'il s'agissait d'une usine de chaudronnerie. (p. 9)

Ce jour-là lempruntant la rue Louis-Blanc], je ne connais pas encore le nom de la rue. (p. 10)

Plus tard je prendrai l'habitude de garer la motocyclette sur un trottoir (p. 14)

Le narrateur fait référence à des «notes * (p. 20), et au fait d'écrire l'histoire du chantier *à la plume" (p. 68). Le texte serait donc

4 Robert Kroetsch, .The Veil of Knowing ", The Lovely Treachery of Words, Toronto/New York/Oxford, Oxford University Press, 1989, p. 187.

5 "Le roman doit être d'abord une "histoire", a story [...] Personnages typés. Enquête plus ou moins policière. Dévoilement d'une cause, d'un ressort, d'un motif, autrement dit d'une culpabilité. Surmontée ou pas, peu importe. Sois coupable, et raconte. [...] Pas de culpabilité, pas de story, ou à peine. * Portrait du joueur, Paris, Gallimard, p. 216. 
une réécriture de l'expérience vécue à travers les photos, puisque le seul "moyen de faire une fin " est de "faire un livre " (p. 81). Le journal intime se présente comme un milieu propre à accueillir des réflexions personnelles ${ }^{6}$, mais également comme un code qui n'est pas entièrement respecté. Ceci invite le lecteur à procéder à un examen minutieux des autres codes.

Les données d'un crime et le profil du criminel peuvent être observés grâce à une grille sociologique. Dans La démolition, nous pouvons aussi parler d'un code sociologique ou d'une critique sociale sous-jacente. Les indices provenant du champ sémantique social s'accumulent; ils apparaissent quelquefois polysémiques. D'abord, la "raison sociale et les coordonnées de l'entreprise " (p. 16) sont notées, et on mentionne la Bastille, lieu historique suggestif (p. 21). Ensuite, les évocations de "l'agit'prop' - (p. 29) et d'une «allégorie du travail, une caricature réaliste-socialiste" (p. 48) sont explicites. Enfin, il est question d'un personnage de roman et de sa * mélancolie petite-bourgeoisen (p. 54). Dans ce contexte, Marianne disparue peut représenter la France (son symbole). La guillotine ainsi que les bombages en trois couleurs (tri-color) invitent à de nouvelles interprétations. Est-ce que ces détails multipliés jusqu'à la provocation sont gratuits, ou sont-ce les signes d'une désintégration de l'idéologie à mettre en parallèle avec une désintégration du langage? Le lecteur-détective est chargé de repérer ce que l'auteur évoque. Pour ce faire, il doit examiner les indices et créer le sens. Il construit une signification à partir du signe vidé de sa substance. La lecture rejoint alors l'écriture.

Les indices qui peuplent ce texte proviennent également du code religieux; ainsi la notion de culpabilité et d'innocence estelle d'abord religieuse. Le narrateur parle de "baptiser le mur" (p. 24) et de la "genèse du chantier * (p. 28). Nous apprenons que * la chute de la cheminée [est] devenue nécessaire pour faire une fin au chantier. (p. 59) et nous savons que le crime (péché originel?) est de "voler le chantier». De plus, le tout se passe immédiatement avant Pâques! Siegfried Kracauer souligne le

6 Dans le roman de Jacques Godbout intitulé Une bistoire américaine (1986), les conventions du roman policier permettent une réflexion sur les valeurs de l'américanéité dans un journal intime écrit a posteriori. Cette réflexion conclut sur le manque d'épaisseur humaine et le manque de culture en Californie. 
parallèle entre le policier et la religion: le mystère, le détectiveprêtre, le criminel-hérétique, et le suspense-ferveur religieuse ${ }^{7}$. Dans ce contexte, le chantier symboliserait le jardin d'Éden du monde industrialisé, et la cheminée représenterait l'arbre. La destruction de la cheminée, métonymie de l'industrie, laisserait un vide dans la grande ville. Le vertige de l'interprétation s'instaure, car les sens possibles ou les pistes suggérées se multiplient.

Les citations bombées constituent un autre élément important; elles font partie du témoignage du texte. Le discours renvoie à un autre discours composé de citations. Dans ce récit donc, différents codes sont présents, mais on ne peut pas décider si l'un d'eux a la prévalence. Cette indécidabilité constituerait le récit même ${ }^{8}$. Toutes les ouvres d'où proviennent les citations sont à mettre en rapport avec le roman policier ou du moins avec le suspense et le mystère. Certaines se réfèrent aussi au problème du langage ou à d'autres textes fictifs 9 . Elles sont tirées de Balzac, Lichtenberg, Chandler, Constant, Gracq, Musset, Stendhal, Godard, Wenders, Perec, Nabokov et autres. Comme le dit Roland Barthes au sujet du popart, el'origine s'efface au profit de la citation .10

L'énonciation passe par les citations qui se transforment en métalangage tentant de décrire ce qui se passe lorsqu'on construit une fiction ${ }^{11}$. La démolition a donc lieu, en un sens, au niveau de

7 Siegfried Kracauer, Le roman policier, Paris, Payot, 1981, p. 16-17.

8 Roland Barthes, L'aventure sémiologique, Paris, Seuil, coll. "Points ", 1985, p. 303.

9 Dans La muse du département, par exemple, l'héroïne de Balzac, Dinah, est sumommee .la Sapho de Saint-Satur "; Sapho, chanteuse, apparaît à la fin de La démolition. De plus, Dinah lit Adolphe pour ne pas commettre les mêmes erreurs qu'Ellénore, par rapport à laquelle elle se définit donc. Le texte de ce roman se réfère aussi à la perte de Beyle (StendhaD. Constant et Stendhal sont cités plus tard dans La démolition. Enfin, nous pouvons parler de la remise en question au moins du signifié lorsque Dinah affirme, a la suite d'un récit de crime, que - bien souvent la victime a été pendant si longtemps le bourreau *. Dans La marquise d'O de Kleist, qui a influencé Kafka, l'intrigue est policière puisque la marquise recherche le père de l'enfant qu'elle porte. L'enfant a été conçu sans qu'elle en ait conscience. Lorsque le père se révèle être celui qui la demande en mariage depuis un certain temps déjà, d'ange qu'il était, cet homme se transforme en démon. Le langage doit encore assumer tous les paradoxes.

10 Roland Barthes, dans Guy Scarpetta, L'impureté, Paris, Grasset, 1985, p. 85.

11 Le métalangage, défini par Barthes, est -un système dont le plan du contenu est constitué lui-même par un système de signification *. Roland Barthes, op. cit., p. 77. 
la fiction pour créer un métalangage cohérent. Linda Hutcheon constate que le roman policier contemporain signale souvent sa propre métafictionalité à travers la fictionalité des conventions qu'il exploite et l'importance qu'il accorde à l'interprétation (dans le processus de l'évaluation du mensonge) ${ }^{12}$.

Le problème de la métafiction et du métalangage nous mène aux couples vrai/faux et fiction/réel ${ }^{13}$. Dès que le narrateur de La démolition parle des "murs de fiction ", l'existence et la nature du mensonge sont posées: les murs font-ils partie de la réalité ou de la fiction? Et les photos des murs? Le texte commente ainsi son essence et sa forme narrative: "J'ai fait tout faux; d'ailleurs, je n'en ai pas la mémoire. (p. 17). À côté des graffiti du narrateur, ce dernier signale "un authentique graffiti dans le plâtre (p. 24). Quel est donc le rapport entre le texte, l'intrigue et les photos? La question de la réalité et de la fiction s'associe intimement au personnage féminin absent de Marianne:

Je me dis qu'il est temps de badigeonner tous ces murs de fiction, afin que Marianne apparaisse bientôt comme une fiction. (p. 30)

Je préfère retrouver celle-ci lune fausse Mariannel, que de chercher l'autre partout ailleurs. (p. 33)

La réalité de Marianne, qui a proprement disparu, passe par une fiction. (p. 68)

S'esquisse alors la tentation de décrire Marianne comme une fiction où tout ne serait pas fictif. (p. 70)

C'est la simulation, comme le note Jean Baudrillard, qui remet en cause la différence vrai / faux. Ce critique affirme que la séduction commence là où les apparences se montrent invulnérables au nihilisme même du sens ou du non-sens ${ }^{14}$. Dans ce texte, la séduction passe par les photos des murs et le personnage absent de Marianne qui créent une tension certaine entre sens et nonsens.

12 Linda Hutcheon, The Canadian Postmodern, Toronto/New York/ Oxford, Oxford University Press, 1988, p. 176.

13 Procbain épisode, le roman d'Hubert Aquin (1965), surdéterminé par les conventions du roman policier, part à la recherche de l'écriture et du méta. Il cherche à donner une signification, à trouver une a fin logique. en se laissant écrire: Je n'écris pas, je suis écrit. Hubert Aquin, Prochain épisode, Montréal Cercle du livre de France, 1965, $174 \mathrm{p}$.

14 Jean Baudrillard, Simulacres et simulation, Paris, Galilée, 1981, p. 231. 
L'intertextualité contribue au flou de la frontière fiction/ réel ${ }^{15}$. Les citations et les références à d'autres textes de fiction connus créent des textes qui circulent et ne renvoient qu'à d'autres textes. Le narrateur évoque Suite vénitienne, le livre qu'il envoie à Marianne. Ce livre de Sophie Calle, photographe (Marianne est aussi photographe), est un texte accompagnant les photos d'une expérience personnelle. L'expérience de la photographe, qui a eu lieu entre le 11 et le 24 février 1980 ( La démolition commence un 7 février), consistait à rechercher et à suivre un homme, Henri B., à travers les rues de Venise pour noter ses déplacements. La photographe parle d'enquête, mais les conventions du roman policier sont bouleversées ou inversées:

Mon enquête se déroule sans lui. Sa découverte risque de tout bouleverser, précipite l'échéance. J'ai peur. (p. 22)

Henri B. n'a rien fait, je n'ai rien découvert. Il fallait une fin banale à cette histoire banale. (p. 57)

Baudrillard, dans sa postface au texte, centre ses remarques sur la question de l'autre: "Filer l'autre, c'est bien lui donner de facto une double vie, une existence parallèle. (p. 85). Mais dans ces circonstances, le double finit par renvoyer à lui-même:

Vous n'existez que dans la trace de l'autre, mais à son insu, en

fait vous suivez votre propre trace, presque à votre insu. [...]

Vous vous séduisez d'être absent, de n'être plus que le miroir de

l'autre qui ne le sait pas. (p. 82)

Dans La démolition, le narrateur se dédouble: "Je ne suis pas le même qui bombe sur les murs et qui fixe les bombages sur la pellicule " (p. 22). Il emploie une stratégie de confession et parle du lien entre "lui et moi * et du rapprochement entre "moi et moi " (p. 29). Il se construit une identité criminelle et un crime. L'écrivain (et ici le narrateur) s'avère le lien entre le détective et le criminel, ainsi qu'entre le photographe et le bombeur.

Le texte de La démolition attire notre attention sur les photos proposées comme référents. Cependant, sur presque toutes les photos, nous trouvons des mots, des signifiants, ou au moins des

15 Le double suspect de Madeleine Monette est à la recherche d'un autre texte, ou d'une vérité, à travers la réécriture d'un premier texte, d'un intertexte inconnu au lecteur. L'essentiel se trouve derrière le non-dit, derrière ce qui est tu, dissimulé. Le texte doit engendrer la vérité. Madeleine Monette, Le double suspect, Montréal, Editions Quinze, 1980, $241 \mathrm{p}$. 
62

lettres. Les graffiti constituent bien sûr les exemples les plus convaincants, mais nous relevons aussi le nom du magasin à l'arrière-plan d'une photo, un sac ou une boîte avec des lettres imprimées (GRP - gouvernement révolutionnaire provisoire?), le nom des démolisseurs sur une pelleteuse, et même une clé plate en forme de " $c$ ".

Dans le roman policier, la tradition veut que le coupable soit le moins suspect en apparence. En un certain sens, dans ce texte, ce sont les mots qui sont les moins suspects: "Ce ne sont que des mots qui pèsent de peu de poids au regard des images que $j$ 'ai faites ". De plus, il existe des inconsistances mineures au niveau des lettres (ou des chiffres), c'est-à-dire au niveau du signifiant: $38 \mathrm{~mm}$ au lieu de 35 (p. 12), un $\mathrm{M}$ double (p. 41), un $\mathrm{H}$ manquant à Hannah (p. 45), un clavier "qwerty" sur lequel le "w" et le * $\mathrm{z}$ * sont intervertis, et enfin une faute d'orthographe sur laquelle le narrateur attire notre attention (p. 55, jeu au pluriel, donc avec un " $X$ ", ce qui rappelle l'expression " $X$ marks the spot ). Le signifiant du mot se décompose, se démolit, et le langage se désintègre. En même temps, ce sont les mots qui sont chargés de perpétuer l'action à travers le bombage de graffiti: "Les bombages en appellent d'autres, jamais innocents, qui s'imposent comme des réponses" (p. 39). Le texte prolifère. Shoshana Felman nous rappelle que le langage possède une nature double: il est référentiel, mais la référence achoppe. Le langage est donc pris en faute. Coupable? ${ }^{16}$

Le langage en jeu ou le jeu du langage est poussé encore plus loin dans la démolition, puisque les citations bombées révèlent souvent la nature sinon ambiguë, du moins polysémique, du langage. Cet "emballement du sens" (p. 40), qui se manifeste de plusieurs façons, atteste l'hyperprésence de l'écriture dans le texte. Dès le début, le narrateur signale sa propre écriture comme écriture: "Sur fond de mur de prison - ce n'est pas une prison, mais ce pourrait. (p. 9-10). La référentialité est niée, sauf comme simulacre. À un autre moment, le rapport signifiant/signifié/

16 Copies conformes, qui respecte les conventions du roman policier, peut être vu comme se rapportant à la recherche de la signification ou de la non-signification du mot amour ", sous le signe de trois épigraphes de Dashiell Hammett à ce sujet. Il s'agit d'une vérité qui nous échappe de plus en plus. Monique LaRue, Copies conformes, Montréal/Paris, Lacombe / Denoël, 1989, 189 p. 
référent est mis en évidence: «Ce fragment de mur où, en haut à droite, dans l'arrondi, est bizarrement gravé dans le plâtre le mot mur. (p. 13). Le langage contient tous les paradoxes et toutes les contradictions: "Je reviens l'après-midi même mettre le mur de Marianne dans la boîte " (p. 24), c'est-à-dire photographier l'inscription "le mur de Marianne". De plus, quelques expressions figées sont déformées. "Conformer * devient "conforter" et un métissage générique est évoqué, au niveau du métalangage, dans la phrase *confortant ainsi aux stéréotypes des deux genres [de bombeur et de photographe] *.

Les citations bombées relèvent presque toutes du jeu du langage sur le langage. En employant les termes de Guy Scarpetta, nous pouvons parler du narcissisme paroxystique de celui qui * exaspère l'artifice, qui joue avec les images qu'il propose, celui derrière lequel toute profondeur disparaît "17. Les mots et les expressions jouent les uns avec les autres. Dans la citation de Stendhal (p. 71), le narrateur commente lui-même le paradoxe d'un personnage qui n'a "rien de naturel * et qui n'est point un "nigaud qui a lu des romans ", alors que les romans sont artifice et contiennent du non-naturel. Il nous invite donc à commenter les autres citations. Dans la première citation du livre, citation de Balzac, ce sont les natures "molles et lâches" qui peuvent "recevoir un nombre infini de blessures" (p. 27), mais la force implique le pouvoir de surmonter les blessures. Dans la citation de Constant (p. 42), l'expression "en un mot" est suivie de plusieurs mots. La citation de Gracq (p. 43) parait tautologique: "Dans la fiction, tout doit être fictif ". La prose de Strauss emploie un négatif complexe et répétitif au lieu d'un négatif simple. L'auteur parle de "renoncer à l'idée de renoncer pour toujours à Hannah" (p. 45). La contradiction semble intrinsèque dans "Au commencement, il y a toujours l'adieu * (p. 78). Il en va de même pour la vie qui est "en couleurs", alors que "le noir et blanc est plus réaliste * (p. 80). Le roman se termine sur la note polysémique et ironique du narrateur qui s'est fracturé le pied: "Demain, lundi, je commencerai ma rééducation. (p. 90).

Comme le note Baudrillard, l'ère de la simulation implique la liquidation de tous les référents, la simulation étant a la gênération par les modèles d'un réel sans origine ni réalité: l'hyperréel " ${ }^{18}$. Le

17 Guy Scarpetta, op. cit., p. 291.

18 Jean Baudrillard, op. cit., p. 10. 
64

crime, dans ce roman, devient crime du signe. Dans la société, le réel est structuré à l'aide du làngage. Tout passe par le langage, qui est notre point de repère; tout est discours. René Girard note que c'est la perte de la différence qui cause une *confusion violente *. Il constate alors que "le langage lui-même est menacé.19. Dans La démolition, c'est l'absence de référent de l'écriture fictive qui est dévoilée. L'auteur écrit dans l'écriture. Nous devons revenir à la citation, car l'originalité n'existe pas: "La littérature est le mode même de l'impossible, puisqu'elle seule peut dire son vide et que, le disant, elle fonde de nouveau une plénitude. "20

Les mots nous échappent et nous n'arrivons pas à leur attribuer un pouvoir de durée. Les signifiés se transforment, car les mots ne sont jamais innocents. En privilégiant le signifiant qui se démolit, le code déconstruit sa propre logique. Les signifiés passent, mais les signifiants, qui sont supposés demeurer ${ }^{21}$, n'y arrivent pas.

Finalement, dans La démolition, le lecteur peut tenter de s'accrocher à sl'évidence. d'un crime, mais ce n'est qu'illusoire. Le texte lui enlève ses repères et il doit rentrer dans le jeu du crimesigne. À travers la désintégration du langage et du signifiant, l'écrivain se met en échec: - Il [l'écrivain] est coupable et toujours bors jeu". Le jeu de l'écriture devient hyperréalité et cri. Marquet-il le triomphe d'une culture vide? Postmoderne? La rééducation du narrateur est sans doute le plan de reconstruction à chercher.

19 René Girard, La violence et le sacré, Paris, Grasset, 1972, p.79: ^Comme dans la tragédie grecque donc, comme dans la religion primitive, ce n'est pas la différence, mais bien sa perte qui cause la confusion violente. La crise jette les hommes dans un affrontement perpétuel qui les prive de tout caractère distinctif, de toute "identité". Le langage luimême est menacé.*

20 Roland Barthes, Essais critiques, Paris, Seuil, 1964, p. 131.

21 Roland Barthes, L'Aventure sémiologique, op. cit., p. 267. 